\title{
ARTÍCULO ORIGINAL \\ Características socio-demográficas y clínicas asociadas a depresión mayor en pacientes con artritis reumatoide
}

\author{
César Pévez-Hernández ${ }^{1,2, a}$, Karina Fiorella Berrocal-Tenorio ${ }^{1, b}$, Blanca Elizabeth Llauca-Ramírez ${ }^{1, b}$, Wendy Ramírez-Quinte ${ }^{1, b}$
}

\author{
Facultad de Medicina, Universidad Nacional San Luis Gonzaga. Ica, Perú 1 \\ Hospital Augusto Hernández Mendoza. Ica, Perú2 \\ Médico reumatólogoa ${ }^{a}$ Médico cirujanob
}

\begin{abstract}
O bjetivo: Identificar las características socio-demográficas y clínicas que influyen en la depresión mayor en pacientes con artritis reumatoide atendidos en el Hospital EsSalud "Augusto Hernández Mendoza" durante los meses de julio a diciembre 2011. Materiales Y Métodos: Se desarrolló un estudio observacional, descriptivo y transversal, que incluyó una muestra de 175 pacientes con artritis reumatoide atendidos en el consultorio de reumatología. Se usaron las pruebas de Chi cuadrado para determinar la relación entre las variables. Se empleó una ficha de recolección de datos, en la que se incluyeron las características socio-demográficas, clínicas de los pacientes. Y el test de Edimburgo. Resultados: La mayoría de los pacientes fueron adultos (135; 77,1\%), de sexo femenino (162; 92,6\%), con antecedente personal $(77,1 \%)$ y familiar $(53,7 \%)$ de depresión, moderado o alto compromiso articular ( $40,57 \%$ y $55,43 \%$, respectivamente). La depresión se halló en 28 (16\%) de ellos y se asoció a la ubicación de la vivienda $(p=0,001)$, el grado de dependencia $(p=0,000)$ y el compromiso articular $(p=0,024)$. Conclusiones: la depresión mayor está presente en los pacientes con artritis reumatoide y se asocia a la zona donde se ubica la vivienda, así como al grado dependencia y el compromiso articular de la enfermedad.

Palabras clave: artritis reumatoide, depresión, dependencia (fuente: DeCS BIREME).
\end{abstract}

Autor corresponsal: Blanca Llauca Ramírez. Dirección: Pasaje Tinguiña Av. San Luis K-13. Ica, Perú. Teléfono: (51) 955967605. Correo electrónico: blanca_Ir7@ hotmail.com

\section{INTRODUCCIÓN}

La artritis reumatoide es una enfermedad articular, sistémica, autoinmune, inflamatoria, crónica y multiorgánica de etiología desconocida $(1,2,3)$. Afecta de forma simétrica y centrípeta las articulaciones sinoviales y produce destrucción progresiva con distintos grados de deformidad e incapacidad funcional $(1,2)$. La principal consecuencia de la artritis reumatoide para el paciente es el deterioro de la calidad de vida, es decir la enfermedad altera el bienestar de las personas en las tres áreas fundamentales del desarrollo físico, mental y social. La capacidad para desarrollar actividades de la vida diaria, tanto físicas como sociales, está considerablemente disminuida en muchos de los pacientes, además la enfermedad tiene importantes repercusiones emocionales (4).

La depresión psíquica constituye un problema de salud pública a escala mundial, hoy cuarta causa de incapacidad y que, según las proyecciones de la Organización Mundial de la Salud (OMS), para el 2020 será la segunda causa más frecuente en los países desarrollados (5).

La depresión clínica es frecuente en pacientes con dolor crónico persistente, el dolor es el síntoma físico más común en pacientes con depresión y un motivo de consulta muy frecuente a los especialistas. El dolor y la depresión se encuentran frecuentemente relacionados y muchos estudios han descrito que los mismos comparten mecanismos neuroquímicos comunes (6).

La depresión sigue siendo un grave problema clínico para casi todos los pacientes con artritis reumatoide, porque sus síntomas traducen fundamentalmente una fuerte asociación con el dolor y, más interesante aún, la posible relación entre depresión y discapacidad. Es bien conocido que la artritis reumatoide representa una de las primeras causas de discapacidad grave en los países desarrollados con las consiguientes pérdidas económicas y sociales (7). De aquellos pacientes que tienen trabajo remunerado al inicio de la artritis reumatoide, $10 \%$ de ellos deja de trabajar dentro del primer año de inicio de la enfermedad y un 50\% están incapacitados después de 10 años(8).

Observamos entonces la importancia de ver al paciente con artritis reumatoide de una forma integral por tal motivo nuestro objetivo es Identificar las características socio-demográficas y clínicas que influyen en la depresión mayor en pacientes con artritis reumatoide, lo que supondría la posibilidad de introducir mejoras tanto en la actuación terapéutica como en la atención al paciente con esta enfermedad.

\section{MATERIALES Y MÉTODOS}

Se realizó un estudio observacional de diseño descriptivo transversal en una población de 370 pacientes con artritis reumatoide atendidos en el consultorio de Reumatología del Hospital IV "Augusto Hernández Mendoza" durante el período julio a diciembre del 2011, de la cual se obtuvo una muestra de 175 pacientes con un nivel de confianza del $95 \%$ y un margen de error del $5 \%$ y un valor para $\mathrm{p}=0.3295$ y $\mathrm{q}=0.6705$, seleccionada mediante muestreo aleatorio simple. Este estudio se realizó previa aprobación en junta médica realizada en el servicio de Medicina.

Se incluyeron hombres y mujeres mayores de 18 años con diagnóstico de artritis reumatoide según los criterios del Colegio Americano de Reumatología. Se excluyeron pacientes: analfabetos, con problemas para 
comunicarse (por hablar otro idioma o tener problemas neurológicos, auditivos o del habla), con enfermedades terminales (cáncer, leucemia, VIH-SIDA o trastornos psiquiátricos conocidos), con tratamiento inmunosupresor o fármacos que induzcan a la depresión (cimetidina, reserpina, neurolépticos, fenotiazídicos), con hipotiroidismo, gestantes o en puerperio inmediato, con antecedente de cirugía mayor reciente, con asociación a otra enfermedad del tejido conectivo, en uso de medicación antidepresiva y/o pacientes que no deseen colaborar con el estudio.

Se empleó una ficha de recolección de datos, en la que se incluyeron las características socio-demográficas y clínicas de los pacientes. Los pacientes fueron captados en los consultorios de Reumatología del Hospital IV "Augusto Hernández Mendoza" en Ica durante los meses julio a diciembre 2011. A cada paciente se le explicó el objetivo del estudio y se le solicitó su aceptación para participar a través de un consentimiento informado escrito y a continuación se le pidió responder las preguntas de la ficha de filiación y de los instrumentos de evaluaciónque mide el grado de actividad de la enfermedad DAS-28 (Disease Activity Score), Cuestionario de Evaluación de Salud, conocido internacionalmente como "HAQ", acrónimo del inglés Health Assessment Questionaire y el test de depresión de Edimburgo para lo cual el paciente marcará los ítems de acuerdo como se siente.

Los datos recolectados, previo control de calidad, fueron ingresados en una base de datos para posteriormente realizar el procesamiento y análisis mediante el uso del programa SPSS 18.0. Se usó la estadística descriptiva para la presentación de frecuencias y porcentajes de las variables categóricas y medidas de tendencia central y dispersión para las variables numéricas. Las variables discretas fueron agrupadas en frecuencias y analizadas con pruebas de chi cuadrado considerando de significancia estadística un valor $\mathrm{p}<0.05$, con una confiabilidad del $95 \%$. Los resultados son presentados en tablas y gráficos haciendo uso del programa MS Word 2010.

\section{RESULTADOS}

Tabla 1, 2 y 3.

\section{DISCUSIÓN}

La artritis reumatoide induce en los pacientes varios procesos interactuantes. Por un lado, la inflamación, el dolor, la pérdida de movimiento articular y la deformidad produce discapacidad. Por el otro, la enfermedad lleva a la pérdida de un gran número de funciones de la vida diaria, lo cual afecta en mayor o menor grado el desplazamiento, el cuidado
Tabla 1. Pacientes con artritis reumatoide según variables clínicas en el consultorio de Reumatología del Hospital IV “Augusto Hernández Mendoza” Essalud- Ica (Julio - Diciembre 2011).

\begin{tabular}{l|ccccc}
\multicolumn{1}{c}{ Variables } & \multicolumn{2}{c}{ Rango } & & \\
\cline { 2 - 3 } & Mínimo & Máximo & Promedio & Error Estándar \\
\hline Tiempo de enfermedad (meses) & 1 & 528 & 141,64 & 9,32 \\
\hline Escala visual análoga del dolor & 2 & 10 & 6,43 & 0,16 \\
Proteína C reactiva & 0 & 57 & 1,83 & 0,37 \\
\hline Factor reumatoide & 1,50 & 798 & 184,29 & 12,20 \\
\hline
\end{tabular}

Tabla 2. Depresión mayor en pacientes con artritis reumatoide según etapa de vida, sexo y lugar de residencia. Consultorio de Reumatología del Hospital IV “Augusto Hernández Mendoza” Essalud- Ica, (Julio - Diciembre 2011).

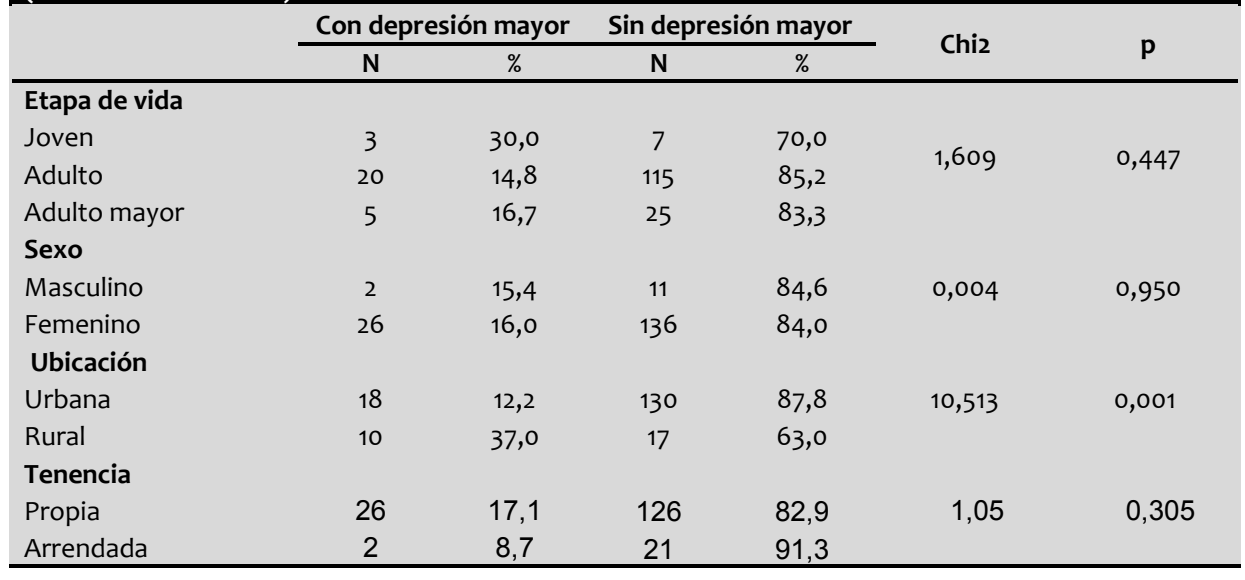

Tabla 3: Depresión mayor en pacientes con artritis reumatoide según características clínicas. Consultorio de reumatología - Hospital IV “Augusto Hernández Mendoza” Essalud-Ica, (Julio - Diciembre

\begin{tabular}{|c|c|c|c|c|c|c|}
\hline & \multicolumn{2}{|c|}{$\begin{array}{c}\text { Con depresión } \\
\text { mayor }\end{array}$} & \multicolumn{2}{|c|}{$\begin{array}{c}\text { Sin depresión } \\
\text { mayor }\end{array}$} & \multirow[t]{2}{*}{ Chi 2} & \multirow[t]{2}{*}{$\mathbf{p}$} \\
\hline & $\mathbf{N}$ & $\%$ & $\mathbf{N}$ & $\%$ & & \\
\hline \multicolumn{7}{|l|}{$\begin{array}{l}\text { Antecedente de } \\
\text { depresión }\end{array}$} \\
\hline Antecedente personal & 23 & 17,0 & 112 & 83,0 & 0,473 & 0,492 \\
\hline Antecedente familiar & 19 & 20,2 & 75 & 79,8 & 2,682 & 0,102 \\
\hline \multicolumn{7}{|l|}{$\begin{array}{l}\text { Progresión de la enfer- } \\
\text { medad }\end{array}$} \\
\hline Estadio I: Temprano & 1 & 11,1 & 8 & 88,9 & 0,479 & 0,920 \\
\hline Estadio II: Moderado & 15 & 16,9 & 74 & 83,1 & & \\
\hline Estadio III: Severa & 11 & 15,1 & 62 & 84,9 & & \\
\hline Estadio IV: Terminal & 1 & 25,0 & 3 & 75,0 & & \\
\hline \multicolumn{7}{|l|}{ Grado de dependencia } \\
\hline Bajo & 9 & 7,3 & 114 & 92,7 & 23,221 & 0,000 \\
\hline Alto & 19 & 36,5 & 33 & 63,5 & & \\
\hline \multicolumn{7}{|l|}{ Compromiso articular } \\
\hline Bajo-moderado & 7 & 9,0 & 71 & 91,0 & 5,168 & 0,024 \\
\hline Alto & 21 & 21,6 & 76 & 78,4 & & \\
\hline
\end{tabular}

personal, el trabajo y otras actividades bási- tos antirreumáticos con drogas inmunosuprecas. Todo ello, altera la independencia econó- soras y citotóxicas, que tienen efectos advermica y los roles sociales de la persona $(9,10)$. Sos frecuentes y potencialmente graves (11).

La depresión en enfermos con artritis reuma- En nuestra serie se halló una frecuencia de toide puede pasar inadvertida fácilmente en $16 \%$. Estos resultados son coincidentes con la atención clínica habitual. El insuficiente ostros estudios que señalan una frecuencia conocimiento sobre la influencia que pueden variable, que va de $15 \% 12$ a $35 \%$ (13).

tener los problemas psiquiátricos en estos Los factores asociados con el desarrollo de enfermos constituye una limitación para se- depresión en distintas series son tener menor leccionar la mejor aproximación terapéutica edad (14), sexo femenino (13), la ausencia de 
pareja (15), y la mayor incapacidad funcional medida por HAQ $(13,16,17)$. Nuestros resultados también demuestran asociación significativa entre la mayor incapacidad funcional o grado de dependencia, así como el compromiso articular de esta enfermedad. Sin embargo, no se halló relación con la edad ni el sexo, posiblemente debido a la mayor frecuencia de pacientes de sexo femenino y adultos o adultos mayores en la muestra estudiada.

Si bien no se ha hallado relación entre la depresión mayor y el tipo de unión conyugal, Cadena y col (18), hallaron que la depresión es frecuente en los pacientes con artritis reumatoide, a pesar de tener un buen entorno familiar, el cual parece actuar ejerciendo un efecto amortiguador en el impacto de la enfermedad sobre la calidad de vida.

Entre las limitaciones, debemos mencionar que no se pudo evaluar el compromiso de otros órganos, aparatos o sistemas debido a que existió dificultad en el acceso a los registros de las ayudas diagnóstica del paciente.

De la investigación se concluye que la depresión mayor se observa en uno de cada dos pacientes siendo más frecuente en el sexo femenino con actividad laboral, la depresión mayor se asocia a la zona donde se ubica la vivienda del paciente y el compromiso articular producto de la enfermedad.

\section{REFERENCIAS BILIOGRÁFICAS}

1. Bermúdez C. Vivir con artritis reumatoide. Sociedad Española de Reumatología. [Internet]. 2009 [acceso 15 de enero del 2011]; 14(2).

2. Tena X, Arnal C, Olivé A. Enfermedades difusas del tejido conjuntivo. En: Rozman C. Medicina Interna Farreras - Rozman. Décimo sexta Edición. Madrid: Elsevier. 2009;10091020

3. Lipski P. Artritis reumatoide. En: Fraga JL. Principios de Medicina Interna. $17^{\mathrm{a}}$ Edición. México. McGraw Hill Interamericana; 2009 (1): 2083-2092.

4. Sociedad Española de Reumatología. Artritis Reumatoide: guía de enfermedad para el paciente. [Internet] Madrid: Jarpyo; 2006. [acceso 7 de enero 2011] Disponible en: http://www.ser.es/ArchivosDESCARGABLES/ Guias_Paciente/Guia_Artritis.PDF

5. Marsiglia I. Estudio clínico y epidemiológico de la ansiedad y la depresión en una muestra de 1159 pacientes. Gac Med Caracas 2010; 118(1):3-10.

6. Scublinsky D. Terapéutica psiquiátrica en fibromialgia, artritis reumatoidea y dolor como síntoma de depresión. Psicofarmacología. 2006; 6(41): 21-29.

7. Yescas E, Ascencio L, Vargas S, et al. Depresión, ansiedad y desesperanza aprendida en pacientes con artritis reumatoide. Psicología y salud. 2008; 18(001):81-89.

8. Ministerio de Salud. Guía Clínica Artritis Reumatoidea. [Internet] Santiago: MINSAL, 2007 [acceso 15 de enero 2011]. Disponible en: http://www.redsalud.gov.cl/archivos/ guiasges/artritispdf.pdf

9. López B, Rincón H, Castellanos J, Gonzáles H. El perfil de minusvalía como la medida de calidad de vida en pacientes reumáticos. Rev Colom Reum. 1993; 1(1):91.

10. Gonzáles H, Moncada J, Badiel M, Badillo Y, Rincón H. Evaluación del programa psicoeducativo para pacientes con artritis reumatoide. Rev Colom Reum . 1995; 2(1):85-86.

11. Velásquez X, Pizarro C, Pizarro P, Massardo L. La depresión en artritis reumatoídea. Reumatología [internet]. 2002; 18(2):49-52. Disponible en: http://www.sochire.cl/bases/r58-1-1343592408.pdf

12. Pincus T, Griffith J, Pearce S, Isenberg D. Prevalence of self-reported depression in patients with rheumatoid arthritis. $\mathrm{Br} J$ Rheumatol. 1996; 35(9):879-883

13. Pastor O, Morales S-V, Llopis G, Ferriol C. Prevalencia y grado de depresión en pacientes con artritis reumatoídea. Med clin [internet] 1998; 111(10):361-366.

14. Wright G, Parker J, Smarr K, Johnson J, Hewett, JE, Walker S. Age, depressive symptoms, and rheumatoid arthritis. Arthritis Rheum. 1998; 41(2):298-305.

15. Abdel-Nasser A, Abd El-Azim S, Taal E, ElBadawy S, Rasker J, Vlakenburg H. Depression and depressive symptoms in rheumatoid arthritis patients: an analysis of their occurrence and determinants. $\mathrm{Br} J$ Rheumatol 1998; 37(4):391-397.

16. Keefe F y Bonk V. Psychosocial assessment of pain in patients having rheumatic diseases. Rheum Dis Clin North Am. 1999; 25(1):81103.

17. Escalante A, del Rincón I, Mulrow CD. Symptoms of depression and psychological distress among hispanics with rheumatoid arthritis. Arthritis Care Res. 2000; 13(3):156167.

18. Cadena J, Cadavid ME, Ocampo MV, Vélez MC, Anaya JM. Depresión y familia en pacientes con artritis reumatoide. Rev Colom Reum. 2002 ;9(3):184-191. 
FINANCIAMIENTO: Autofinanciado

CONTRIBUCIONES DE AUTORÍA: CPH, KFBT, BELR Y WRQ participaron en el diseño del estudio, el análisis de los datos, revisaron críticamente el artículo y aprobaron la versión final.

\title{
Sociodemographic and clinical associated with depression in patients with rheumatoid arthritis
}

\begin{abstract}
bjective: To Identify the socio-demographic characteristics and clinical variables that influence the greater depression in patients with rheumatoid arthritis treated at the Hospital EsSalud "Augusto Hernández Mendoza" during the months of July to December 2011. Materials and Methods: It was developed an observational, descriptive and transversal research,. which included a sample of 175 patients with rheumatoid arthritis were treated at the clinic of rheumatology. We used the Chi-square test to determine the relationship between the variables. Employment is a tab of data collection, which included the sociodemographic characteristics, clinical patients. And the test of Edinburgh. Results: The majority of the patients were adults (135; $77.1 \%)$, female (162; $92.6 \%$ ), with personal history $(77.1 \%)$ and family $(53.7 \%)$ of depression, moderate or high commitment articulate ( $40.57 \%$ and $55.43 \%$, respectively). The depression was found in $28(16 \%)$ of them and was associated with the location of the housing $(p=0.001)$, the degree of dependency $(p=0.000)$ and the articular involvement $(p=0.024)$.Conclusions: Major depression is present in patients with rheumatoid arthritis and is associated with the area where the housing, as well as the degree unit and the joint involvement of the disease.

Key words: Rheumatoid arthritis, depression, dependence.
\end{abstract}

\section{CITA.}

Pévez-Hernández C, Berrocal-Tenorio KF, Llauca-Ramírez BE, Ramírez-Quinte W. Características sociodemográficas y clínicas asociadas a depresión mayor en pacientes con artritis reumatoide. Rev méd panacea.2013; 3 (1):15-18. 